\title{
Digital servitization: How data-driven services drive transformation
}

\author{
Christian Kowalkowski \\ Linköping University \\ christian.kowalkowski@liu.se
}

Alexey Sklyar

Linköping University

alexey.sklyar@liu.se

\author{
Bård Tronvoll \\ Inland Norway University \\ bard.tronvoll@inn.no
}

\author{
David Sörhammar \\ Stockholm University \\ david.sorhammar@sbs.su.se
}

\begin{abstract}
The infusion of data-driven services in manufacturing provides new opportunities for longterm competitive advantage; however, it also poses new challenges and entails tradeoffs among strategic options. Digital servitization changes intra-firm processes and customer relationships as well as overall ecosystem dynamics. Drawing on an extensive study of ABB Marine \& Ports, a market-leading systems integrator, the concept of digital servitization is examined by analyzing its key characteristics, including opportunities and challenges for manufacturers. The resource integration patterns that connect actors and the dual role of technology in both increasing resource integration complexity and in facilitating the coordination of complexity are discussed. Advancing digital servitization requires fostering service-centricity and executing strategic change initiatives for both the internal organization and the broader ecosystem. Firms must undertake three interlinked changes: (1) digital, (2) organizational, and (3) ecosystem transformations. In addition to contributing to the service literature, these findings provide actionable insights for managers.
\end{abstract}

\section{Introduction}

The infusion of data-driven services into an increasing number of aspects of business illustrates that digital transformation and servitization are closely intertwined. For manufacturing firms, digital servitization provides new opportunities for long-term competitive advantage; however, it also poses new challenges because it blurs industry boundaries and alters the established market positions of firms. In addition, digital servitization changes intra-firm processes and customer relationships as well as overall ecosystem dynamics [1].

Although manufacturers are strategically investing in data collection, analytics capabilities, and cloud-based platforms, many firms remain concerned about how to best address digital disruption and enable digitalization [2]. The COVID-19 pandemic has accelerated this change and placed additional pressure on firms; unless a firm can transform accordingly, it risks being outpaced by competitors [3]. Despite being a central topic for most firms, little information exists to guide firms' actions in structuring operations for digitalization [4]. As Raddats et al. [5] point out, increasing knowledge about how manufacturing firms manage digital servitization is a key research priority.

This transformation extends beyond the individual firm [6] to encompass resource integration between actors embedded in larger structures as elements of a service ecosystem [7]. Resource integration refers to how actors cocreate a contextspecific, uniquely determined value for themselves and other actors in the ecosystem [8]. As digital technology has become a critical facilitator of value co-creation [9], it has changed resource integration patterns and has ultimately transformed service ecosystems [10]. Technologies have also become increasingly autonomous, impacting resource integration among people, organizations, and things [11]. The rapid and disruptive nature of technological change makes it vital for manufacturers to reconfigure their resource integration patterns, both in their strategic interests and for the viability of the overall ecosystem.

Against this backdrop, the organizational transformations that underpin digital servitization as well as the underlying resource integration patterns among ecosystem actors are examined. Based on an extensive study of a market-leading systems integrator 
pursuing a digitalization strategy-ABB Marine \& Ports - three major interlinked changes have been revealed, and how transformation within the service ecosystem increases the importance of software and data-driven services in interactions between firms has been determined. The findings also show the dual role of technology in increasing the complexity of resource integration and in facilitating the coordination of this complexity. To take full advantage of digitalization beyond purely technological benefits, firms must foster service-centricity and execute strategic change initiatives for the internal organization and the broader ecosystem.

\section{Theoretical background}

\subsection{Digital servitization}

Servitization refers to a firm's transition from a product-centric business model and logic that focuses on selling products to a more service-oriented business model and logic that focuses on facilitating customer value creation through advanced services and solutions [5, 12]. By its very nature, service requires more intense and closer customer interactions that facilitate connections at different organizational levels and that help a firm acquire a better understanding of its customers' businesses and needs as well as those of its customers' customers [13]. Merging the physical and digital worlds has become an emerging research area within the servitization domain under the term digital servitization, which we define as the utilization of digital technologies for transformational processes from a product-centric to a service-centric business model and logic.

There is a difference between digitization, which involves turning analog data into digital data [14], and digitalization, which refers to the use of digital technology to provide new value-creating and revenue-generating opportunities (i.e., to change a business model) [15]. To be successful, a firm must manage digitalization, which includes the sociotechnical processes that accompany digitization [16]. Drawing on the concept of the resource-based view [17], this implies that a firm can achieve a competitive advantage by developing and deploying digital resources and capabilities; however, as Ulaga and Reinartz [6, p. 6] point out, "resources per se do not confer competitive advantage but must be transformed into capabilities to do so." For example, the acquisition of installed base product usage and process data is a necessary but not sufficient condition for service-related data processing and interpretation capability. Thus, the acquisition of strategic customer data is a necessary but not sufficient condition for servitization [6]. As digital business opportunities are rapidly increasing, the challenge is seldom to develop or acquire the required resources but rather to have the ability to integrate them, meaning being able to deploy the digital capabilities needed [18].

Furthermore, digital technology can be a doubleedged sword for servitization. A study by Perks et al. [19] illustrates that many manufacturers have explored technical possibilities (e.g., remote connectivity) without having a clear service business model in mind. Rather than truly understanding the customer's business needs and how to conduct value-based selling based on enhanced customer performance, it is tempting to either mimic what competitors are doing or to provide a service for free with the hope that customers would eventually discover (some of) its value and be willing to pay for it; however, as the connected installed base grows and the costs of collecting and managing data increase each year, it becomes increasingly difficult to defend such a technology-centric approach unless service sales begin to materialize. By providing services free of charge, the perceived value of the service offering is reduced from the customer's perspective [20].

Hsuan et al. [21] characterize servitization as a system comprising product and service systems and digital servitization as a system comprising servitization and software systems. Each system can be further decomposed into architectures and can be conceptualized in a polar fashion: product (modular versus integral), service (basic versus advanced), and software (open versus proprietary platforms). Digital servitization creates complexity in relationships among ecosystem actors, leading to increased coordination costs and risks [22], conflicting authority structures, and an increased demand for platforms that can overcome these challenges [23]. Due to the increased complexity, digital servitization also increases the governance-related challenge of balancing control and flexibility [24, 25].

Coreynen et al. [26] distinguish between servitization that encompass "back-end" and "frontend" digitalization. Essentially, back-end digitalization relates to company-specific aspects, whereas front-end digitalization can utilize either an open or a proprietary software architecture [21] and relates to inter-firm aspects, such as changing ecosystem relationships and configurations [27]. From a back-end perspective, digital servitization can enhance operational efficiency, facilitating a closer integration between units. From a front-end perspective, it can enable new forms of customer interactions and a closer integration with network actors [26]. Evidence suggests that in addition to influencing existing relationships, digital servitization 
can enable new resource configurations (i.e., resource integration patterns) [28] that affect relationships both internally and in the broader ecosystem (e.g., [29]). As some accounts of digital servitization suggest, a disruption of existing configurations may negatively affect interactions between actors [30].

\subsection{Resource integration in service ecosystems}

Service ecosystems increasingly serve as the context and unit of analysis for value co-creation and resource integration [7]. A service ecosystem can be defined as a "relatively self-contained, self-adjusting system of resource integrating actors connected by shared institutional arrangements and mutual value creation through service exchange" [31, p. 161]. This service ecosystem perspective provides a systemic, dynamic, and contextual understanding [32]. This is critical in understanding the complexity of digitalization in service ecosystems because the structure drives behaviors within the system, and any shift in the underlying rules of the system can serve as a powerful point of leverage for change [33].

To create a competitive advantage through digital servitization, digital resources (digitization) as well as the capabilities to implement and transform them (digitalization) are needed. Resource integration, which is how actors co-create value [8], is also a key capability in a digital context. Digital technology functions as a critical facilitator of value creation because it modifies resource integration patterns that connect ecosystem actors between and within organizations [34]. Whereas the resource-based view of the firm (e.g., [17]) tends to focus on resources that are controlled within the firm, this ecosystem perspective acknowledges that to various degrees, a single actor is dependent on resources controlled by other actors for a competitive advantage as well as for the viability of the ecosystem as a whole. The rapid and potentially disruptive nature of technological change makes it critical for manufacturers to be able to adapt and alter their resource integration patterns when pursuing digital servitization [35].

\subsection{Strong and weak ties}

Resource integration within an ecosystem occurs between strongly and weakly tied actors. Actors within ecosystems generally become strongly tied over time as they learn to draw on each other's heterogeneous resources more efficiently and effectively. The more the actors mutually adapt their processes and routines, the stronger the ties between them become [36]. Adaptation and a close relationship are generally viewed as prerequisites for the provision of complex services and customer solutions [37]; however, in pursuing digital servitization, manufacturers may encounter a paradox: mutual adaptation and resources that bring success in the traditional product domain may become core rigidities that emerge within the preexisting network of ties, thus constraining the transformation effort. In particular, strongly tied patterns of resource integration make it more difficult to adapt to technology-driven environmental changes [38], leading to resource integration that simultaneously constrains change (e.g., by inhibiting the adoption of new technologies).

While strongly tied actors integrate more resources than weakly tied actors, the rigidities formed by such institutionalization may inhibit more extensive change and even create resistance ("incumbent inertia" [38]). In such circumstances, to acquire novel resources beyond those already available (e.g., data science skills), a firm may have to interact with weakly tied actors [36]. Therefore, weak ties may play an important role in ecosystem transformation and change, such as digital servitization.

\subsection{Technology as an operant resource}

Technology has been described as both an operand resource (facilitator or enabler) and an operant resource (initiator or actor) in value creation [16]. Vargo and Lusch [39] define operand resources as resources on which an operation or act is performed to produce an effect. They compare them with operant resources, which are employed to act on operand resources (and other operant resources). Technological advances mean that machines, technologies, and other resources previously considered operand are now increasingly capable of adjusting to their environment as operant resources [16]. By viewing technology as an operant resource, actors can extend their ability to reconfigure resource integration patterns within the ecosystem, such as in information technology's capacity to enable and facilitate knowledge sharing and coordination [40].

Unlike strongly tied ecosystem actors, weakly tied actors are more likely to perceive new properties as a resource, such as when exploring the potential to separate and transport information independently of people and materials [41]. Weakly tied actors can also visualize how the same resource can be recombined (or unbundled and rebundled [41]) into new resource integration patterns. In contrast, a service ecosystem involving strongly linked actors with well-defined institutional rules may not perceive these possibilities. While weakly linked actors can see the operant 
properties of new technologies, strongly linked incumbent firms tend to perceive these technologies as an operand resource on which an act is performed [16]. In this sense, technologies and their resource value are socially constructed [42, 43] by institutional rules within the ecosystem.

The core rigidities of strongly tied actors create institutionalized rules that determine the meaning of certain resources, which resources to integrate, and how to best integrate the resources. When the service ecosystem is undisturbed by disruptive technology, it conforms to Alderson's [44] idea of a perfectly heterogeneous market in which actors possess and exchange unique resources, and a resource's value depends on how it is integrated with other resources and on how the integration is perceived by the beneficiary [31]; however, in cases of technologydriven environmental change, the ecosystem's institutionalized rules may inhibit change or even blind actors to the potential use-value of the new or altered resource. The concept of change is of direct concern to incumbent firms seeking to adjust resource integration patterns in the service ecosystem. From a service ecosystem perspective [7], change is ongoing as each resource integration activity creates potential change for all operand and operant resources. This highlights that ecosystem actors can change and adapt their resource integration patterns and that technology, which is increasingly viewed as an operant resource, contributes to this transformation.

\section{Research method}

\subsection{Research setting}

To understand the ability of firms and networks to digitalize their service operations, we adopted a discovery-oriented, theory-in-use approach $[6,37]$ to carry out a longitudinal in-depth single case study [45] spanning over 14 years. The case firm-ABB Marine \& Ports - and its network were actively studied over 2.5 years, while the preceding events were examined exclusively through secondary data. Digital servitization has been actively progressing throughout the explored period (2006-2020) with profound effects on the involved actors. A qualitative approach with a focus on insights from managers allowed for transparent observations [45] of a revelatory case [46]. Directly applicable to the studied case of digital servitization, this approach also enabled a contextspecific understanding of the processual nature of an organizational transformation and of the underlying dynamics of phenomena that play out over time.

We utilized a theoretical sampling approach for case selection based on three criteria: (1) to acquire data for theory building, we chose a firm that had been undergoing a strategic move toward digitalization and service-led growth; (2) to avoid speculative futureoriented insights, we selected a firm that was actively and strategically investing in digital servitization; and (3) we chose a case that would provide access to both real-time and retrospective data [47] through key informants across functions and hierarchical levels as well as through secondary sources. The selected case firm is a leading provider of maritime solutions for large multinational vessel owners and operators.

\subsection{Data collection and analysis}

Discussions of data collection began in December 2015 when we requested permission to investigate digital servitization at the case firm. In total, in-depth interviews with 33 executives and managers involved in the firm's transformation strategy and execution were conducted between May 2016 and June 2020, with the interview duration spanning 0.5-3.5 hours. The respondents were selected through snowball sampling [48] and social networking sites, such as LinkedIn. Key informants were interviewed on more than one occasion if additional questions emerged, resulting in 12 additional interviews. Using a semistructured interview guide, we aimed to obtain a comprehensive understanding of digital servitization to identify the changes occurring in the service ecosystem as it moved from a pre-digitalized to a digitalized state. In particular, we were interested in developing an in-depth understanding of organizational shifts and underlying resource integration mechanisms between actors in the ecosystem. Primary sources were comprised of meeting observations and visits to digital service centers, while secondary sources included annual reports and internal documentation as well as company magazines and websites. Data collection reached saturation when no new insights emerged.

In total, the interview transcripts yielded over 700 pages of single-spaced text. Once saturation was achieved, transcripts, notes, and secondary data were read and coded to identify key issues and themes using NVivo software . Coding was based on a comparative content analysis supported by peer evaluations and on three main criteria [6, 37]: (1) whether an insight could be considered applicable beyond a specific context; (2) whether an insight was provided by several informants; and (3) whether an insight concerned not just obvious but also interesting and useful information. All the researchers who collected the data also participated in coding for an independent parallel analysis and triangulation [46]. The process involved reading the interview transcripts and field notes and 
then comparing and interpreting each record, facilitating re-extraction, and re-coding data based on discussions between the researchers.

\section{Findings and discussion}

Drawing on an extensive study of ABB Marine \& Ports, a market-leading systems integrator in the maritime industry, the findings point to four drivers of digital servitization: the growing role of platformbased business models, the adoption of recurring revenue models, the shift from frontline-heavy field services to back office-heavy data-driven services, and the growing emphasis on embedding sustainability goals into corporate strategies. First, based on its software platform, ABB offers its customers cloudbased services, including applications developed by third-party providers. Second, the firm was innovating by increasingly focusing on subscription models with recurring revenues. Third, as more service activities can be performed onshore through digital service centers, such as remotely supporting customer staff on vessels, the role of traditional field services has been transformed. Finally, ABB collaborates with customers to improve the overall environmental performance of its operations through various advisory services. These changes have major implications for the way the firm organizes its operations and engages with customers.

As ABB pursued digital servitization, it moved from a pre-digitalized to a digitalized state, which affects the digital solutions, the firm, and the ecosystem. We identified three interconnected transformations in facilitating the shifts as the firm moved between the two states: digital, organizational, and ecosystem transformations. In addition, the findings demonstrate the role of technology in enabling actors to successfully coordinate and manage the complexity of resource integration patterns. As digital, data-driven services have become central to resource integration, the integration has become increasingly effective through technological support for connectivity and continuous interaction and the related need for a more extensive coordination of resource integration activities.

\subsection{Digital transformation}

Whereas the majority of the maritime industry's players began their transformational processes in recent years, ABB's digital servitization spanned over a decade. Initially, the firm transformed itself throughout 2006-2009 to become a systems integrator with a focus on obtaining knowledge of customer operations. The latter became a key enabler for developing the firm's first digital services when ABB concentrated its R\&D efforts on both the service and technology aspects of novel offerings. Simultaneously, the firm began hiring employees who would be more "open" to new technology, such as statisticians and business analytics, thus further enabling digital servitization. Developed specifically for ABB's installed base, the first digital services were closely connected to the hardware of the firm and its partners. For example, digital services for remote diagnostics developed at the time were aimed at monitoring and troubleshooting the firm's equipment.

In 2010, ABB began to extend its digital services to third-party hardware, which resulted in a significant competitive advantage over the subsequent years. In addition to upgrading the already existing digital services, new offerings became available, such as a digital service aimed at monitoring the fuel and energy consumption of the vessels for the optimization of energy flows. Parallel to extending its digital service portfolio, the firm initiated changes in back-office processes to support digital servitization.

Digitalization became prominent in the previously conservative maritime industry around 2014, when customers began viewing data ownership as a critical issue. ABB launched a comprehensive digital servitization initiative that emphasized the interconnected nature of its services resulting from digital servitization. The wide-ranging initiative involved integrating a critical infrastructure, productizing digital services as modular offerings, unifying software-related businesses, and large-scale hiring of employees with skills in digitalization.

By 2019, ABB had an extensive platform-based service portfolio and a wide range of subscriptionbased services, including remote diagnostics and advisory services. In contrast to established subscription models in consumer markets, the managers found it challenging to standardize and scale up these data-driven services given the heterogeneity of vessel configurations and customer types. Some customers requested "cyber resilient vessels," so the firm expanded its portfolio of cybersecurity services. The firm also emphasized promoting the digital servitization initiative to its customers.

Overall, the described findings regarding digital transformation point to the importance of dematerialization - the separation between data and physical manifestations. In the pre-digitalized stage, high-quality performance data were generally scarce; however, in the digitalized stage, ongoing dematerialization has created an abundance of data. The shift was facilitated by data centricity and the seizing of data-related opportunities. Specifically, the firm developed new digital capabilities, such as for 
mediating interactions between actors via digital technology (and thus eliminating the inter-actor isolation) as well as for recombining sets of longitudinal and multi-source data to achieve a competitive advantage. Thus, the firm became able to develop and provide new services, such as data-driven advisory and cybersecurity services. To do so, a new set of employees had to be recruited (e.g., computer scientists and data analysts), which led to a more diverse set of human resources. For instance, growing concerns among customers about cybersecurity accelerated the firm's decision to hire employees with data science and cyber security skills.

\subsection{Organizational transformation}

To move from a pre-digitalized to a digitalized ecosystem state, a firm must make two interrelated organizational shifts: from planning to discovery and from hierarchy to partnership. The first shift relates to a firm's identity and self-perception of its business model. The case firm transformed from a more traditional planning-oriented identity to a more discovery-oriented one and defined itself as a digital technology company. Legitimization and agility facilitated this shift. Legitimization mechanisms facilitated digital servitization for both internal and external actors. For example, the firm provided a vision for transformation, envisioned how it and its customers would operate in the future, and invited customers to their new digital operations centers to experience the new software-centric services firsthand. This emphasis on visionary leadership are also vital for traditional servitization, although the specific focus here is the digital facets of the change. Another key component was to meet the demands of agility linked to digital servitization, which required changing some practices and elements of the organizational culture to cope with faster software development lifecycles. This included standardizing and formalizing customer-specific solutions and striving for greater scalability (i.e., productizing). The entrepreneurial mindset and culture within the firm helped drive the change.

Fostering collaboration was the second shift, which was required to break the silo mentality and to move from hierarchy to partnership. Multi-actor coupling, which refers to the joint activities of ecosystem actors, and reciprocal value propositions with clear benefits for both parties facilitated the change. Digital services required a much less physical presence of the firm's employees onboard customer vessels, but to take advantage of this benefit, collaborative learning was required to improve the knowledge and skills of the customer staff. The abundance of data drew the firm closer to many of its customers operationally as well as strategically. Furthermore, the firm had to foster collaboration between its different units, including both its traditional front-end and back-end units, and its new digital entity. While there was concern among some executives that digital services would cannibalize the firm's established service business, the resistance decreased as the firm placed specific emphasis on gaining a profound knowledge of customers' businesses to craft value propositions with clear benefits for both parties. To develop more competitive value propositions, it became vital to assess the digital maturity of each customer.

\subsection{Ecosystem transformation}

In the pre-digitalized ecosystem, the focus of servitization initiatives was on hardware rather than software, resulting in limited scalability across customer segments and a restriction on the extent to which third-party actors could connect and integrate. Due to the lack of a digital infrastructure that would enable real-time connectivity, actors typically interacted through analog or one-way digital communication, such as email. During the 2010s, the firm's ecosystem changed into what we refer to as a digitalized state. The firm acted as a network orchestrator assembling and managing an interorganizational network to achieve a collective goal [49], which drove the change. Rapid technological development enabled the key actors in the ecosystem to build the digital infrastructure needed for continuous real-time connectivity, which resulted in digital technology meditating all interactions among the actors. Examples include onshore operations centers and customized user portals and interfaces supported by third-party cloud services. Importantly, the interaction between onshore and offshore units, which had traditionally been (relatively) isolated, was enhanced, and simultaneous remote access for both the captain and the chief engineer enhanced decisionmaking processes. From having a secondary and supporting role in the pre-digitalized ecosystem, digital technology (such as enabling telecommunication between shore and ship) became critical to interactions in the digitalized stage as well as the key selection criterion when fleet owners and operators selected equipment suppliers. The new digital infrastructure also enabled scalability and thirdparty compatibility, which would not have been possible to achieve in the previous stage of the transformation.

In terms of resource integration, our findings shed light on major differences between pre-digitalized and 


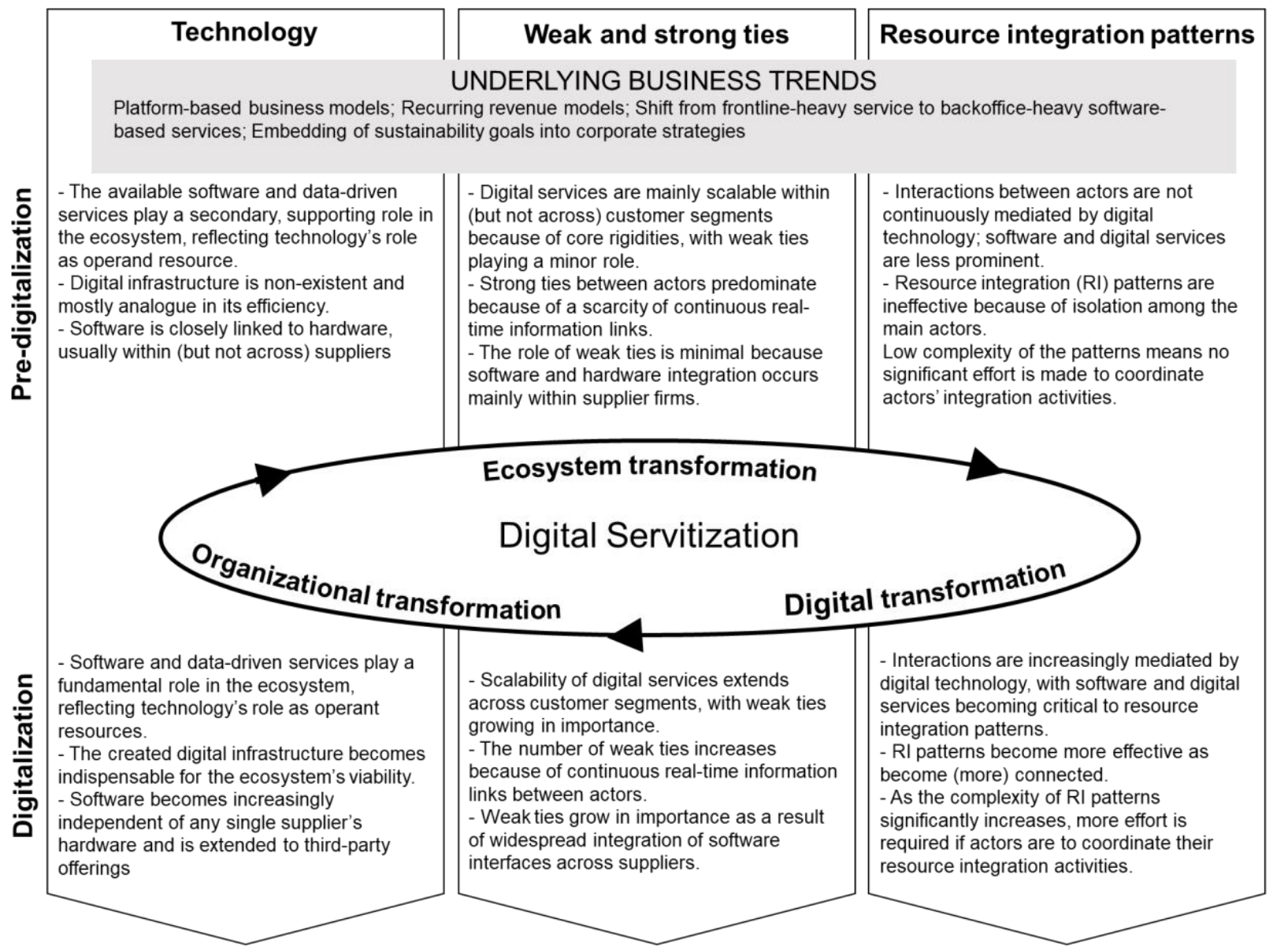

\section{Figure 1: The transformations of digital servitization}

digitalized ecosystems in terms of (1) interplay between technology, (2) strong and weak ties, and (3) resource integration patterns. In the pre-digitalized state, ecosystem actors largely depended on nonresource integration patterns; however, the dependence on strong ties could create core rigidities [50], resulting in less effective continuous resource communication and strong ties dominated integration patterns. The secondary supporting role in the ecosystem reflected technology's role as an operand resource, meaning a resource on which an act is performed [16]. The digitalized state allowed weaker ties to play a central role in the mediation of interactions among actors. A case in point is the onboard operations of vessels that could be connected to onshore operations for actors who had previously been disconnected (i.e., weak ties). As digital technologies facilitate such weakly tied interactions, new resource integration patterns emerged, which involved more spatially dispersed actors and enabled more effective resource integration patterns. This change reflected technology's extended role as an operant resource, or a resource that produces effects.
Examples of new data-driven services based on satellite-based connections include more accurate weather forecasts, digitalized ship engine optimizations tools, and online shipping navigation systems.

\section{Implications for research and practice}

Manufacturing firms generally struggle to innovate and exploit data-driven services. Figure 1 summarizes the characteristics of the transformations required to achieve digital servitization and to move from a pre-digitalized to a digitalized ecosystem based on transformations in digital technology, organization, and the ecosystem. These transformations are enabled through a shift from scarcity to abundance regarding data, from planning to discovery within the organization, and from hierarchy to partnership with ecosystem actors. By analyzing the successful transformation of a legacy manufacturer in a dynamic industry, we contribute to the digitalization, service, and managerial practice literature. 


\subsection{Theoretical implications}

Theoretically, the case illuminates the importance of extending the view of digital servitization from a rather narrow view of technology to three interlinked processes: digital transformation, organizational transformation, and ecosystem transformation.

A digital transformation is enabled by focusing on the technological affordance [51] and having a changed mindset regarding technology. Due to this new posited impact of digitalization on resource integration patterns, technology should be considered an operant rather than an operand resource. This change reveals a clear distinction between the predigitalized and digitalized states. It radically changed interactions between actors within the service ecosystem; technology proved critical in enhancing resource-integrating patterns within the service ecosystem by becoming the facilitating "actor" that coordinated these interactions. Thus, it improved the overall effectiveness of the way resources are integrated into both existing and new service offerings. By perceiving technology as an operant resource, the technology itself plays a dual role. It increases the complexity of resource integration patterns and simultaneously enables the engaged ecosystem actors to better manage this complexity.

Digital servitization also demands a change in each participating organization's business logic. The case shows that to become digital, the firm must adjust its organizational identity and culture. While these are not new insights-previous research addresses the need for greater clarity [52]-prior studies have mainly focused on obstacles associated with organizational identity and culture when transforming to digital servitization. The present research empirically illustrates how a focal firm can successfully change its identity and culture, especially by adjusting the balance between its strongly and weakly connected counterparts.

Moreover, a transformation to digital servitization for an incumbent firm can hardly be managed singlehandedly by a firm. Rather, it requires a transformation of the whole ecosystem in which the firm operates. This requires that decision makers view the firm as part of a system, where a change in their resource integrating pattern needs to be cocreated, or at least accepted, by all engaged actors. To enhance ecosystem viability, our findings suggest that other actors in the ecosystem must undergo a similar, albeit not identical, transformation. Hence, digital servitization requires changes in the entire network (service ecosystem) from a systems perspective, which illustrates how social and economic actors cocreate value in specific contexts.

\subsection{Managerial implications}

At a practical level, our findings show that digital services alone are not enough to achieve effective resource integration due to the low levels of digital maturity and strong ties among established actors in the pre-digitalized ecosystem. A comprehensive digital infrastructure had to be implemented to establish many weak ties, enabling more effective resource integration patterns. As in many other industries and ecosystems, intra-firm and inter-firm core rigidities inhibited the changes needed to drive digital servitization. In this case, better connectivity and information exchange improved decision making and increased the transparency of operations within and among ecosystem actors. The digital infrastructure helped managers respond to environmental changes and take advantage of further technological advancements, creating a competitive advantage for the individual firm and the ecosystem as a whole.

To drive the transformation, management should develop a credible and captivating vision for its key customers and partners. To shift from vision and strategy to realization, the firm needs commitment from these stakeholders and new types of employees, such as data scientists, which means increased competition with the IT industry (and other manufacturers) for talent. In this rivalry, the firm is expected to also face the demands of agility from the fast-paced developments in the IT realm. As a result, changing the organizational culture and aligning it with the transforming workforce becomes critical for digital servitization. While servitization is traditionally viewed as an incremental and emergent process, the digital side of the change requires a more purposeful and coordinated effort. In addition, the shift to back office-heavy automation and software-based services implies significant changes both for the service organization and the customer-provider interfaces.

Finally, managers must acknowledge that competition in the digital domain may be fundamentally different from traditional servitization, such as spare parts provision and field services. As we observed, customers increasingly seek providers able to integrate systems and provide a uniform platformwhether open or proprietary-beyond traditional product and industry categories. Thus, competition may come from various software and hardware companies as well as established incumbent manufacturers. Therefore, ensuring the compatibility of digital services with offerings from other firms can bring substantial benefits. For instance, if the firm extensively interconnects its services with third-party hardware and software, the resulting data-driven 
benefits are likely to prove highly valuable for the customers. Overall, regardless of industry and service maturity, manufacturing firms must transform using digital servitization to build and sustain a competitive advantage.

\subsection{Limitations and further research}

As with any qualitative inquiry, this study is limited in its capability to support empirical generalizations. The single case study design means that the results can only be generalized theoretically [45]. While the findings are transferable to other industrial ecosystems, especially those based on complex product systems, larger-scale qualitative and quantitative studies should test the validity of our findings. For example, offshore and onshore premises as well as the complexity of vessels (e.g., product, service, and software integration) make the maritime industry different from industries with an installed base on customer premises (e.g., industrial robots or warehouse trucks). Furthermore, research shows that culture plays a central role in digital transformation, especially for services. While an incumbent, the case firm is known for its entrepreneurial culture, which helped drive the change according to several respondents. Future research should study cases that do not display this idiosyncrasy.

\section{References}

[1] Jovanovic, M., D.R. Sjödin, and V. Parida, Coevolution of platform architecture, platform services, and platform governance: Expanding the platform value of industrial digital platforms. Technovation 2021. in press.

[2] Björkdahl, J., Strategies for Digitalization in Manufacturing Firms. California Management Review, 2020. 62(4): p. 17-36.

[3] Rapaccini, M., et al., Navigating disruptive crises through service-led growth: The impact of COVID19 on Italian manufacturing firms. Industrial Marketing Management, 2020. 88: p. 225-237.

[4] Zeithaml, V.A., et al., A Theories-in-Use Approach to Building Marketing Theory. Journal of Marketing, 2020. 84(1): p. 32-51.

[5] Raddats, C., et al., Servitization: A contemporary thematic review of four major research streams. Industrial Marketing Management, 2019. 83: p. $207-$ 223.

[6] Ulaga, W. and W.J. Reinartz, Hybrid Offerings: How Manufacturing Firms Combine Goods and Services Successfully. Journal of Marketing, 2011. 75(6): p. 5-23.
[7] Vargo, S.L. and R.F. Lusch, Institutions and axioms: An extension and update of servicedominant logic. Journal of the Academy of Marketing Science, 2016. 44(1): p. 5-23.

[8] Kleinaltenkamp, M., et al., Resource integration. Marketing Theory, 2012. 12(2): p. 201-205.

[9] Balaji, M.S. and S.K. Roy, Value co-creation with Internet of things technology in the retail industry. Journal of Marketing Management, 2017. 33(1/2): p. 7-31.

[10] Storbacka, K., et al., Actor engagement as a microfoundation for value co-creation. Journal of Business Research, 2016. 69(8): p. 3008-3017.

[11] Breidbach, C., et al., Operating without operations: how is technology changing the role of the firm? Journal of Service Management, 2018. 29(5): p. 809-833.

[12] Baines, T. and H.W. Lightfoot, Servitization of the manufacturing firm: Exploring the operations practices and technologies that deliver advanced services. International Journal of Operations \& Production Management, 2014. 34: p. 2-35.

[13] Kowalkowski, C. and W. Ulaga, Service Strategy in Action: A Practical Guide for Growing Your B2B Service and Solution Business. 2017.

[14] Ng, I.C.L. and S.Y.L. Wakenshaw, The Internetof-Things: Review and research directions. International Journal of Research in Marketing, 2017. 34(1): p. 3-21.

[15] Svahn, F., L. Mathiassen, and R. Lindgren, Embracing digital innovation in incumbent firms: How Volvo cars managed competing concerns. MIS Quarterly, 2017. 41(1): p. 239-254.

[16] Lusch, R.F. and S. Nambisan, Service Innovation: A Service-Dominant Logic Perspective. MIS Quarterly, 2015. 39(1): p. 155-176.

[17] Barney, J.B., Firm Resources and Sustained Competitive Advantage. Journal of Management, 1991. 17(1): p. 99-120.

[18] Kamalaldin, A., et al., Configuring ecosystem strategies for digitally enabled process innovation: A framework for equipment suppliers in the process industries. Technovation, 2021. in press.

[19] Perks, H., et al., Network orchestration for value platform development. Industrial Marketing Management, 2017. 67: p. 106-121.

[20] Ulaga, W. and S. Michel, Bill It, Kill It, or Keep It Free? MIT Sloan Management Review, 2019. 60(2): p. 45-49.

[21] Hsuan, J., M. Jovanovic, and D.H. Clemente, Exploring digital servitization trajectories within product-service-software space. International Journal of Operations \& Production Management, 2021. in press. 
[22] Suppatvech, C., J. Godsell, and S. Day, The roles of internet of things technology in enabling servitized business models: A systematic literature review. Industrial Marketing Management, 2019. 82: p. 7086.

[23] Eloranta, V. and T. Turunen, Platforms in servicedriven manufacturing: Leveraging complexity by connecting, sharing, and integrating. Industrial Marketing Management, 2016. 55: p. 178-186.

[24] Kamalaldin, A., et al., Transforming providercustomer relationships in digital servitization: A relational view on digitalization. Industrial Marketing Management, 2020. 89: p. 306-325.

[25] Vendrell-Herrero, F., O.F. Bustinza, and M. Opazo-Basaez, Information technologies and product-service innovation: The moderating role of service R\&D team structure. Journal of Business Research, 2021. 128: p. 673-687.

[26] Coreynen, W., P. Matthyssens, and W. Van Bockhaven, Boosting servitization through digitization: Pathways and dynamic resource configurations for manufacturers. Industrial Marketing Management, 2017. 60: p. 42-53.

[27] Struyf, B., et al., Toward a multilevel perspective on digital servitization. International Journal of Operations \& Production Management, 2021. In press.

[28] Story, V.M., et al., Capabilities for advanced services: A multi-actor perspective. Industrial Marketing Management, 2017. 60: p. 54-68.

[29] Parida, V., P. Oghazi, and S. Cedergren, A study of how ICT capabilities can influence dynamic capabilities. Journal of Enterprise Information Management, 2016. 29(2): p. 179-201.

[30] Vendrell-Herrero, F., et al., Servitization, digitization and supply chain interdependency. Industrial Marketing Management, 2017. 60: p. 6981.

[31] Lusch, R.F. and S.L. Vargo, Service-Dominant Logic: Premises, Perspectives, Possibilities. 2014, Cambridge, UK: Cambridge University Press.

[32] Chandler, J.D., et al., How Does Innovation Emerge in a Service Ecosystem? Journal of Service Research, 2019. 22(1): p. 75-89.

[33] Meadows, D.H. and D. Wright, Thinking in systems : a primer. 2008, Chelsea Green Pub.: White River Junction, Vt.

[34] Sklyar, A., et al., Organizing for digital servitization: A service ecosystem perspective. Journal of Business Research, 2019. 104: p. 450-460.

[35] Sklyar, A., et al., Resource integration through digitalisation: a service ecosystem perspective. Journal of Marketing Management, 2019. 35(11/12): p. 974-991.
[36] Granovetter, M., The strength of weak ties: A network theory revisited. Sociological Theory, 1983: p. 201.

[37] Tuli, K.R., A.K. Kohli, and S.G. Bharadwaj, Rethinking Customer Solutions: From Product Bundles to Relational Processes. Journal of Marketing, 2007. 71(3): p. 1-17.

[38] Lieberman, M.B. and D.B. Montgomery, FirstMover Advantages. Strategic Management Journal (John Wiley \& Sons, Inc.), 1988. 9: p. 41-58.

[39] Vargo, S.L. and R.F. Lusch, Evolving to a New Dominant Logic for Marketing. Journal of Marketing, 2004. 68(1): p. 1-17.

[40] Nambisan, S., Information Technology and Product/Service Innovation, 2013. 14(4): p. 215-226.

[41] Normann, R., Reframing business : when the map changes the landscape 2001, Chichester: Wiley

[42] Pinch, T.J. and W.E. Bijker, The social construction of facts and artefacts. Social studies of science, 1984: p. 399-441.

[43] Wieland, H., N. Hartmann, and S. Vargo, Business models as service strategy. Journal of the Academy of Marketing Science, 2017. 45(6): p. 925943.

[44] Alderson, W., Dynamic marketing behavior: a functionalist theory of marketing. 1965, Homewood, IL: Irwin. IX, $383 \mathrm{~s}$.

[45] Yin, R.K., Case study research : design and methods. 4th ed. ed. Applied social research methods series. Vol. vol. 5. 2009, Thousand Oaks, Calif: Sage.

[46] Bryman, A. and E. Bell, Business research methods. 4th ed. ed. 2015, Oxford: Oxford University Press.

[47] Pettigrew, A.M., Longitudinal Field Research on Change: Theory and Practice. Organization Science, 1990. 1(3): p. 267-292.

[48] Coleman, J., Relational Analysis: The Study of Social Organizations with Survey Methods. Human organization, 1958. 17(4): p. 28-36.

[49] Paquin, R.L. and J. Howard-Grenville, Blind Dates and Arranged Marriages: Longitudinal Processes of Network Orchestration. Organization Studies, 2013. 34(11): p. 1623-1653.

[50] Leonard-Barton, D., Core Capabilities and Core Rigidities. Strategic Management Journal, 1992. 13: p. 111-125.

[51] Nambisan, S., et al., Digital Innovation Management: Reinventing Innovation Management Research in a Digital World. MIS Quarterly, 2017. 41(1): p. 223-238.

[52] Kohtamäki, M., et al., Digital servitization business models in ecosystems: A theory of the firm. Journal of Business Research, 2019. 104: p. 380-392. 